\title{
Organophosphate Pesticide Exposure and Glucose Homeostasis
}

\author{
Mya Thanda Sein* and Mya Pwint Phyu \\ Department of Physiology, University of Medicine,Magway, The Republic of Union of Myanmar
}

*Corresponding author: Mya Thanda Sein, Professor and Head, Department of Physiology, University of Medicine, Magway, The Republic of Union of Myanmar

\section{ARTICLE INFO}

Received: 彗January 30, 2019

Published: 慧 February 08, 2019

Citation: Mya Thanda Sein, Mya Pwint Phyu. Organophosphate Pesticide Exposure and Glucose Homeostasis. Biomed J Sci \& Tech Res 14(2)2019. BJSTR. MS.ID.002525.

\section{ABSTRACT}

Present review discusses the organophosphate (OP) pesticides toxicity with associated hyperglycemia in animal as well as human studies and effect of OP pesticide on liver enzymes involved in glucose homeostasis pathways. Pancreas keeps hormonal control of glucose homeostasis by secretion of glucagon and insulin. Insulin resistance is higher risk of impair glucose homeostasis. Based on cumulative evidences, oxidative stress induced by OP pesticides may lead to disturbance of glucose homeostasis. There is increasing interest in environment pesticides exposure can affect the glucose homeostasis.

\section{Introduction}

Organophosphate (OP) compounds have been used as pesticides since 1975. OP insecticide compounds are a diverse group of chemicals. This compound contains different group of chemicals such as acephate, diazinon, dimethorate, parathion, phosmet, malathion, parathion, diazinon, fenthion, dichlorvos, chlorpyrifos, ethion, azamethiphos [1]. There is emerging evidence that OP has various toxicities such as neurotoxicity, endocrine toxicity, immunotoxicity, reproductive toxicity, genotoxicity and cellular oxidative imbalance and impaired glucose homeostasis at the hazard of human and animal health. It was found the notable coincidence of occurrences in association between OP poisoning and severe hyperglycemia. Two patients, mother and son presented with many complicated symptoms including hyperglycemia and administration of pralidoxime (AChE activator) normalized blood glucose level. Though acetylcholinesterase enzyme level had not been detected in these patients, they had history of exposure to malathion sprayed in their area [2]. Many case report studies had described the detail sign and symptoms of OP pesticide poisoning accompanying hyperglycemia. Then, it has been raised awareness of association between OP poisoning and severe hyperglycemia in human.

\section{Effect of OP Pesticide on Liver Enzymes Involved in Glucose Homeostasis Pathways}

Culminating hyperglycemia has been increasingly reported as outcome of OP poisoned animal model studies. Exposure of rats to OP compounds such as Malathion [3-5], acephate [6] and dimethoate [7] developed hyperglycemia with stimulation of hepatic gluconeogenesis and glycogenolysis. The possible mechanism of increase blood glucose level might be due to stimulation of hepatic gluconeogenesis and glycogenolysis for increased energy production to detoxification. Upon entering the body- through contact with skin and mucous membrane, inhalation and ingestion- OP pesticides avidly binds the AChE by forming covalent bond between OP pesticides and oxygen of serine at the active site of AChE then it transforms into irreversible phosphorylated inactive AChE and lead to increased acetylcholine activity $[8,9]$. Thus, hyperesthesia, intermittent spasm, muscular tremors, sustained seizures and muscle fasciculation are induced by cholinergic action of OP pesticides [10]. These involuntary energy demanding activities trigger the release of glucose by glycogenolysis and gluconeogenesis. It also stimulates the glycolysis of liver and muscle, and subsequent releases of ATP to meet the body's energy requirement $[4,11]$.

\section{Effect of OP Pesticide Exposure on Oxidative Stress}

Exposure to OP pesticides, inhibits the AChE enzyme and increases the activity of muscarinic type of Ach receptor. The overstimulation of Ach receptors result in uncoordinated nerve and muscle stimulation leading to sustained seizures and muscle fasciculation [10]. During these conditions, the flow of oxygen through brain and muscle is greatly increased. This metabolic stress 
results in increase requirement the ATPs for them and increase demand of glucose, which in turn activates the glycolysis. Glycolysis is oxygen independent metabolic pathway that converts glucose into pyruvate. This process releases the high energy molecule ATP (adenosine triphosphate) and NADH (reduced nicotinamide adenine dinucleotide) [12,13]. These products of glycolysis are oxidized by using atmospheric oxygen.

$$
\mathrm{NADH} \stackrel{\text { Oxidation }}{\longrightarrow} N A D^{+}+H^{+}+2 e^{-}
$$

According to redox reaction, oxidation is a process in which an atom or a group of atoms taking part in chemical reaction loses one or more electrons and remain an uneven number of electrons. An atom or group of atom with an even number of electron (unpaired electron) is also known as free radical (ROS). Rapid release of ROS due to increase metabolism is called respiratory burst. Respiratory burst activates cell membrane-bound enzyme NADPH oxidase (NOX) which catalyzes one-electron reduction of oxygen to superoxide anion, and the process involves oxidization of cytosolic NADPH to NADP and free radicle (ROS).

$$
\left.\mathrm{NADPH}+\mathrm{H}^{+}+2 \mathrm{O}_{2} \rightarrow \mathrm{NADP^{+ }}+2 \mathrm{H}^{+}+2 \mathrm{O}_{2^{-}} \text {( a free radical }\right)
$$

\section{Oxidative Stress and Insulin Resistance}

Insulin is the major hormone controlling glucose metabolism. When the blood glucose level is increased, glucose stimulates insulin secretion from beta cell of pancreas. When glucose enters the beta cell, glucose is metabolized, and ATP was generated. Then, closure of ATP sensitive $\mathrm{K}^{+}$channel increases intracellular $\mathrm{K}^{+}$concentration and cause the membrane depolarization. And then opening of voltage-gated $\mathrm{Ca}^{2+}$ channels open and increased $\mathrm{Ca}^{2+}$ concentration in cytoplasm causes activation of protein kinase and release of insulin by exocytosis $[14,15]$. When insulin binds to insulin receptor $\alpha$ subunits, there is autophosphorylation of $\beta$ subunits on tyrosine residues and activates the insulin receptor tyrosine kinase, which phosphorylates and recruits different substrate adaptors such as the Insulin receptor substrate (IRS) family of proteins. Tyrosine phosphorylated IRS, then displays binding sites for phosphatidylinositol 3- kinase (PI3-kinase). PI3kinase then phosphorylates phosphatidylinositol 2- kinase (PIP2) to PIP3 which in turn activate protein kinase B (Akt), eventually leading to glucose transporter 4 (GLUT4) translocation to plasma membrane of skeletal muscle cells and adipocyte, thus allowing the cells to absorb extracellular glucose, lowering plasma glucose level.

Ma et al. proposed the mechanism about the oxidative stress and insulin resistance [16]. During the oxidative stress, NADPH oxidase 4 (NOX4) is a powerful oxidizing enzyme that produces ROS. In this condition, there was a shift in the signaling pathway that happens at PI3-kinase. PI3-kinase phosphorylates Rac GTP ase instead of PIP2, which amplifies the activity of NOX4 [17]. The increased ROS activates casein kinase-2 (CK2) which in turn activates the retromer. The retromer then signals the trans-Golgi network downstream, and GLUT4 is transported to lysosomes for degradation instead of the plasma membrane. Therefore, intravascular glucose levels remain elevated and cause the insulin resistance.

\section{Conclusion}

The anticholinesterase effects of OP insecticides are produced by the binding of the phosphate groups of this chemicals compound to the AchE that hydrolyzes acetylcholine (Ach) neurotransmitter at the neuromuscular junction. Excessive accumulation of Ach at cholinergic site produce nicotinic effects including intermittent spasm, muscular tremors, sustained seizures and muscle fasciculation resulting from prolonge continuous stimulation of Ach neurotransmitter at motor endplate. Thus, oxygen and glucose demand as well as ATP requirement are greatly increased throughout the muscles. After that, the glycolysis pathway is activated to achieve the increase energy requirement and NADH as a product of glycolysis pathway is oxidized by using atmospheric oxygen resulting in increased production of free radical (ROS). These ROS shift the insulin signaling pathway at PI3-kinase to phosphorylate the Rac GTPase leading to degradation of the GLUT4 in lysosome. Thus, intravascular glucose levels remain elevated and cause the insulin resistance.

\section{References}

1. Kamel F, Hoppin JA (2004) Association of pesticide exposure with neurologic dysfunction and disease. Environ Health Perspe 112(9): 950.

2. Meller D, FraserI, Kryger M (1981) Hyperglycemia in anticholinesterase poisoning. CMAJ 124(6): 745-748.

3. Gupta PK (1974) Malathion induced biochemical changes in rats. BCPT 35(3): 191-194.

4. Abdollahi M, Donyavi M, Pournourmohammadi S, Saadat M (2004) Hyperglycemia associated with increased hepatic glycogen phosphorylase and phosphoenolpyruvatecarboxykinase in rats following subchronic exposure to malathion. CBP Toxicology \& Pharmacology137(4): 343-347.

5. Lasram MM, Annabi AB, Rezg R, Elj N, Slimen S, et al. (2008) Effect of short-time malathion administration on glucose homeostasis in Wistar rat. Pesticide Biochemistry and Physiology 92(3): 114-119.

6. Deotare ST, Chakrabarti CH (1981) Effect of acephate (orthene) on tissue levels of thiamine, pyruvic acid, lactic acid, glycogen and blood sugar. Indian J Physiol Pharmacol 25(3): 259-264.

7. Reena K, Ajay K, Sharma CB (1989) Haematological changes induced by dimethoate in rat. Arhiv Za Higijenu Rada I Toksikologiju 40(1): 23-27.

8. Namba T, Nolte CT, Jackrel J, Grob D (1971) Poisoning due to organophosphate insecticides: acute and chronic manifestations. The American Journal of Medicine 50(4): 475-492.

9. Haddad LM, Shannon MW, Winchester JF (1983) Clinical Management of Poisoning and Drug Overdose 3 Aufl, Saunders, Philadelphia, USA.

10. Costa LG (2006) Current issues in organophosphate toxicology. Clinica Chemica Acta 366(1-2): 1-13.

11. Sarin, S, Gill KD (1999) Dichlorvos induced alterations in glucose homeostasis: possible implications on the state of neuronal function in rats. Molecular and Cellular Biochemistry 199(1-2): 87-92.

12. Joseph N, Zhang-James Y, Perl A, Faraone SV (2015) Oxidative stress and ADHD: a meta-analysis. Journal of Attention Disorders 19(11): 915-924.

13. Halliwell B (2007) Oxidative stress and cancer: have we moved forward? Biochemical Journal 401(1): 1-11. 
14. Cooper KH (2013) Aerobics program for total well-being: Exercise, diet, and emotional balance. Bantam.

15. Hançer NJ, Qiu W, Cherella C, Li Y, Copps KD, et al. (2014) Insulin and metabolic stress stimulate multisite serine/threonine phosphorylation of insulin receptor substrate 1 and inhibit tyrosine phosphorylation. JBC 289(18): 12467-12484.

\section{ISSN: 2574-1241}

DOI: 10.26717.BJSTR.2019.14.002525

Mya Thanda Sein. Biomed J Sci \& Tech Res

(C) This work is licensed under Creative

Submission Link: https://biomedres.us/submit-manuscript.php
16. Ma J, Nakagawa Y, Kojima I, Shibata H (2014) Prolonged insulin stimulation down-regulates GLUT4 through oxidative stress-mediated retromer inhibition by a protein kinase CK2-dependent mechanism in 3T3-L1 adipocytes. JBC 289(1): 133-142.

17. Hurrle S, Hsu WH (2017) The etiology of oxidative stress in insulin resistance. Biomedical journal 40(5): 257-262.

\begin{tabular}{ll} 
BIOMEDICAL & Assets of Publishing with us \\
RESEARCHES & - Global archiving of articles \\
& - Immediate, unrestricted online access \\
\hline
\end{tabular}

